\title{
Adaptive Q-aware Scheduling Algorithm for Multi- Service Flows in 802.16j Networks
}

\author{
Rama Reddy $\mathrm{T}$. \\ G B R PG College \\ Anaparthi(AP), India
}

\author{
Satya Prasad R. \\ Nagarjuna University \\ Guntur, India
}

\author{
Prasad Reddy \\ P.V.G.D. \\ Andhra University \\ Visakhapatnam, \\ India
}

\author{
Pallamsetty S. \\ Andhra University \\ Visakhapatnam, India
}

\begin{abstract}
The IEEE 802.16j standard defines Mobile Multi-hop Relay Wireless Cellular Networks. Introduction of Relays in WiMAX networks pose new challenges along with the advantages of High Throughput and Improved Coverage Area. Thus Scheduling plays a crucial role in Mobile Multihop Relay(MMR) Wireless Networks. The Queue aware scheduling algorithm [1] maximizes the throughput by considering Queues at Base Station(BS) and Relay Station(RS) as well along with Concurrent Transmission Technique. In Queue aware scheduling, as all the arrived packets are queued up in FCFS manner, the high priority packets are obviously delayed. In this paper, QOS guarantee is incorporated by considering multiple Queues for different service flows at Base Station for Queue aware scheduling algorithm. Real Time Adaptive Scheme (RTAS) [2] is modified to improve throughput and used for picking the packets from service class sub queues(SCSQ) to include them in BS input queue. The results show that high priority packets are given proper importance while considering low priority packets as well. Throughput is also increased because of Modified RTAS and Concurrent Transmission Technique of Queue aware scheduling.
\end{abstract}

\section{General Terms}

WiMAX, Scheduling, Algorithm, Throughput, Queue, Packet

\section{Keywords}

IEEE 802.16j, Base Station, Mobile Stations, Relay Stations, QOS, RTAS, MRTAS, Packet Delay

\section{INTRODUCTION}

Mobile Multi-hop Relay WiMAX(Wireless interoperability for Microwave Access) Networks based on IEEE 802.16j[3] standard provides Long Range High Speed Broadband Cellular Services than its predecessor IEEE 802.16e[4]. The main advantages of $802.16 \mathrm{j}$ compared to other wireless technologies like 802.11[5], are the longer transmission range, higher throughput and more sophisticated support for QoS at the MAC level. With single Base Station(BS) as the only serving station, the range of the cell should be smaller to provide high quality service to the Mobile Stations(MS) at the cell edge, as the capacity of the link varies with range[6], hence is limited. To increase the cell coverage or to improve the throughput in the cell coverage or both, in a cost effective way, the concept of Relays are introduced in 802.16j standard. As the Scheduling of frames at MAC level was not defined in the standard and is left open for discussion, various schemes were proposed for Down Link(DL) or for Up Link(UL) or for both[7][8][9][10]. These algorithms did not considered Queues at Relay Stations. The Queue aware scheduling algorithm [1] focused the current Queue sizes of Base
Station(BS) as well as Relay Stations(RSs) and achieved high throughput with reasonable fairness in Mobile Multi-hop Relay (MMR) wireless cellular networks. Here, the BS maintains a Main Queue(BSMQ) for each Mobile Station connected to it directly or indirectly(thru one or more RSs). All the incoming packets destined to the connected Mobile Stations are queued by the BS in FCFS manner in their respective BSMQ. Then the packets are scheduled by Queue aware scheduling algorithm, after finding the Concurrent Transmission Scenarios(CTS) by the Greedy Algorithm by considering the high back pressure links to enhance the overall network throughput. By considering current queues at BS and Relay Stations, the goal of achieving high throughput is reached, but at the same time high priority packet are also treated normally and hence suffering from unwanted delay.

IEEE 802.16 standard specified QoS parameters at MAC layer by dividing the service flows into five different classes, like UGS, ertPS, rtPS, nrtPS and BE. Generally the UGS and ertPS traffic packets are originated by the VOIP applications without silence suppression and with silence suppression respectively, rtPS packets come from real time video applications, nrtPS packets are originated by file transfer applications and BE packets are from low priority applications like email and net browsing.

To treat the packets according to their priorities, a two level integrated scheduling scheme is proposed in this paper. In the first level, Classifier Module(CM) prepares Service Class Sub Queues(SCSQ), then the modified real time adaptive scheme(MRTAS) based on RTAS [2] executes to bring the specified Quantity of packets based on the Sub Class Quantum value. In the second level, the Queue aware scheduling algorithm is executed to schedule the prioritized packets so as to increase the overall network throughput by considering CTS with high back pressure. Simulation results showed that the modified RTAS integration with Queue aware scheduling is best suited in providing QoS guaranteed service with high throughput achievement.

The rest of the paper is organized as follows. Section II discusses the related work. Section III explains the QoS importance and high priority packets delay problem in Qaware Scheduling, Real Time Adaptive Scheme and Modified RTAS to improve the throughput and proposed integration of Modified RTAS with Queue aware scheduling. Simulation results are provided in Section IV and concludes the paper in Section V.

\section{RELATED WORK}

In this section some proposed scheduling algorithms related to WiMAX networks are presented. In [11] a scheduling algorithm was proposed for $802.16 \mathrm{j}$, where directional 
antennas are used to exploit the spatial reuse in an urban area with very high obstructions. They proposed a scheduling algorithm to enhance network throughput, but the process to determine concurrent transmission scenarios in relay based networks is not addressed. In [12] a multi-hop wireless network with in-band relay is considered. The RSs accumulate traffic for its connected MSs. A linear programming model is used to calculate the minimum time to transmit a fixed data load from BS to every RS over possibly multiple hops. Though the throughput is maximized, they neither consider the queue size at RS, nor consider the framebased feature of WiMAX networks. [1] considers the varying traffic load(Q size) of each RS and also considers the framebased feature of WiMAX networks. In this paper, the authors focused on maximizing the overall network throughput by identifying Concurrent Transmission Scenarios efficiently but have not considered the QoS requirements of various Service Flows, so the high priority packets suffer from unwanted delay because of low priority packets.

In [7] the authors proposed a packet scheduling scheme that comprises of two levels. In the first level, a deficit fair priority queuing (DFPQ) mechanism is proposed in order to schedule the whole bandwidth to the different class of services. In the second level, earliest deadline first (EDF) is used for rtPS service flows, weight fair queuing (WFQ) is used to schedule nrtPS and round robin (RR) is used for BE service flows. In [8] strict priority service is applied in the first level, where as the second level is almost similar to [7].Because of the strict priority in [8] some high priority connections starve bandwidth. The above discussed schedulers didn't pay attention to queue size and probably consider infinite queue lengths, authors in [2] propose Real Time Adaptive Scheme(RTAS) to use finite queue sizes specially for real-time service flows like UGS and rtPS. With a proposed simple modification to RTAS the overall throughput can be improved by increasing the Deficit Counter(DC) of nrtPS service flows when the real time service flows are not congested. To combine the benefits of Queue aware scheduling algorithm and modified RTAS, a two level integration scheme is proposed to achieve high throughput while considering the different service flows and their priorities.

\section{ADAPTIVE Q-AWARE SCHEDULING FOR 802.16J NETWORKS WITH FOCUS ON QOS}

In [1], the authors presented a Queue aware scheduling algorithm in the form of Linear Programming Problem in multi-hop relay networks, and also discussed a Greedy Algorithm to find Concurrent Transmission Scenarios(CTS) in relay networks which are part of the input to LP problem. Throughput is increased because of the Concurrent Transmission mechanism and delay is also increased because of the Common Queues for all the service flows.

\subsection{Queue aware Scheduling Advantages and Drawbacks}

Figure. 1 illustrates the architecture a simple Relay Based Network of IEEE 802.16j [2] with Queue aware scheduling only. BS connects to RS and/or MS, and each RS can connect further to other RS and/or MS. Base Station and Relay Stations maintain one Queue per Mobile Station connected to it directly or indirectly. In Queue aware scheduling, BS needs to collect the downlink real time queue size of its associated RS and this queue information is sent to BS using uplink bandwidth. The resulting signaling change due to uplink queue status report is insignificant, and the corresponding uplink bandwidth consumption is negligible. After gathering RS queue information, BS runs the scheduling algorithm to obtain the downlink scheduling results and broadcasts the results to RS and MS. In this centralized approach, the BS has full control of its associated RS and MS. The signaling and architecture change due to the introduction of RS is limited. The presence of RS is transparent to MS hence no changes required in MS, and the signaling change of reporting RS queue size is restricted between RS and BS. Hence this centralized approach is a feasible solution and is easy to implement with minor changes in BS. As specified in [1], CTS is a set of all links that can transmit at the same time, i.e., no two links in a CTS share the same transmitter or receiver.

\section{Some Typical Challenges in Scheduling:}

1. As the input for the scheduling algorithm, concurrent transmission scenarios need to be determined in an efficient way. When adding a link candidate into a concurrent transmission scenario, it must be guaranteed that adding this link will not decrease the total throughput of this scenario. However, it is not practical to traverse all possible links searching for concurrent scenarios due to the non-linear growth of links with respect to number of MS and RS.

2. Due to the fact that wireless cellular networks are framebased, and the corresponding scheduling algorithm must take this factor into consideration. In each frame, different concurrent scenarios must share this frame duration. Thus arises the issue of fair allocation of time resources among various MS who share one frame, while still achieving the goal of maximum network throughput.

3. Let the scheduling algorithm adjust to the real-time queue size change in RS.

4. Provide prioritized service to all the users based on their requirements in the Network, which needs the consideration of QoS(service flows) in the system.

Queue aware scheduling successfully faced the above three challenges except the QoS consideration.

Advantage: Because of the simultaneous transmissions the throughput is drastically increased in Queue aware scheduling.

Drawback: At the same time the delay is also increased to high priority service flows.

Reason: The BS maintains a separate queue for each MS as shown in figure 1. All the incoming (downlink) packets are queued up from different service flows into the common queue of the corresponding MS in FCFS manner as shown in figure 1.

\subsubsection{QoS Specifications of Different Service Flows:}

As specified in IEEE 802.16j [2], five different service flows are supported in the mobile version of WiMAX as shown in Table 1: Unsolicited Grant Service (UGS), Extended RealTime Polling Service (ertPS), Real-Time Polling Service 




Fig 1: Architecture of Relay Based Network with Q-aware scheduling

(rtPS), non real-time polling service (nrtPS), and Best Effort (BE). Each of these scheduling services has a mandatory set of QoS parameters.

Table 1. WiMAX applications and QoS specifications

\begin{tabular}{|c|c|c|}
\hline QoS Category & Applications & QoS Specifications \\
\hline UGS & VOIP & $\begin{array}{l}\text { - Maximum Sustained Rate } \\
\text { - Max. Latency Tolerance } \\
\text {-Jitter Tolerance }\end{array}$ \\
\hline ertPS & $\begin{array}{l}\text { Voice with } \\
\text { Activity } \\
\text { Detection } \\
\text { (VoIP) }\end{array}$ & $\begin{array}{l}\text {-Minimum Reserved Rate } \\
\text {-Maximum Sustained Rate } \\
\text {-Max. Latency Tolerance } \\
\text {-Jitter Tolerance } \\
\text {-Traffic Priority }\end{array}$ \\
\hline $\mathrm{rtPS}$ & $\begin{array}{l}\text { Streaming } \\
\text { Audio or } \\
\text { Video }\end{array}$ & $\begin{array}{l}\text {-Minimum Reserved Rate } \\
\text {-Maximum Sustained Rate } \\
\text {-Max. Latency Tolerance } \\
\text {-Traffic Priority }\end{array}$ \\
\hline nrtPS & $\begin{array}{l}\text { File Transfer } \\
\text { Protocol } \\
\text { (FTP) }\end{array}$ & $\begin{array}{l}\text {-Minimum Reserved Rate } \\
\text {-Maximum Sustained Rate } \\
\text {-Traffic Priority }\end{array}$ \\
\hline $\mathrm{BE}$ & $\begin{array}{c}\text { E-mail, Web } \\
\text { Browsing }\end{array}$ & $\begin{array}{l}\text {-Maximum Sustained Rate } \\
\text {-Traffic Priority }\end{array}$ \\
\hline
\end{tabular}

\subsection{RTAS-A Dynamic Scheduling Scheme}

The Real Time Adaptive Scheduling(RTAS) algorithm is based on the Deficit Round Robin (DRR) concept [13], where a quantum of service $(\mathrm{Q})$ is assigned to each sub queue that decides the number of packets to be served into the frame in each round. Corresponding Deficit Counters (DC) are used in order to keep track of deficit queues that have to be compensated in the next round.
The scheduler is composed of five sub queues which correspond to the five service flows. For rtPS, packet's deadline must be considered by the scheduler. This implies that arrived packets should be served without missing their deadlines and those with urgent deadline should be served in priority. Thus, rtPS connections are scheduled upon arriving in the rtPS sub-queue using the Earliest Deadline First (EDF) scheduling mechanism [14], while First Come First Serve (FCFS) policy is applied for UGS, ertPS, nrtPS and BE priority queues.

Real-time data rate of a service flow can be observed by the change of size of the corresponding priority queue.

While queue size for non real-time traffic can be considerably large, delay sensitive class of services should have a relatively smaller queue size. Expected steady state queue length will not be calculated here, instead the instantaneous queue length is considered which, in reality, reflects the real-time data rate.

The scheduler needs to know the quantum value $\left(Q^{t}\right)$, the deficit counter(DC) and the queue length $\left(\mathrm{q}^{\mathrm{t}}\right)$ for each priority queue, in order to determine how many data packets from each class will be served in each round. In [2], the authors proposed simple equations to calculate a quantum value that is more adapted to the required QoS and real-time characteristics of each class of service in a multi-service network like WiMAX.

The equations used to calculate quantum values based on queue sizes of the priority queues of UGS, ertPS, rtPS, nrtPS and $\mathrm{BE}$ are given below.

Adaptive or real time quantum value $\mathrm{Q}^{\mathrm{t}}$ is calculated at every $M$ period of time, where $M(=n * f d)$, is at least one frame duration (fd).

\subsubsection{Quantum values for UGS and ertPS:}

UGS and ertPS service flows are allocated a fixed bandwidth grant in each TDD frame. It is assumed that all arrived UGS packets in the current frame will be served in the next frame, 
and thus its quantum value (Qugs) can be calculated as follows:

$$
\begin{gathered}
\mathrm{Q}_{\text {ugs }}=\sum_{\mathrm{i}=\mathbf{0}}^{\mathrm{N}} \mathrm{R}_{\max } \\
\mathrm{Q}_{\text {ert }}=\sum_{\mathrm{i}=\mathbf{0}}^{\mathrm{N}} \mathrm{R}_{\max }
\end{gathered}
$$

Where $\mathrm{N}$ refers to the maximum number of active UGS/ertPS connections, and Rmax is the maximum sustained rate.

\subsubsection{Quantum values for rtPS and nrtPS :}

$\mathrm{Q}^{\mathrm{t}}=\mathrm{Q}^{\mathrm{t}^{\mathrm{t}}}(1+\alpha)$ where $\alpha=\sum\left(\mathrm{q}^{\mathrm{t}}-\mathrm{q}^{\mathrm{t}^{\prime}}\right) / \mathrm{q}^{\mathrm{t}}, \alpha$ is the total queue variation ratio over $\mathrm{M}$ period

For rtPS service flows,

$$
\mathrm{Q}_{\mathrm{rt}}(\min )=\sum_{\mathrm{i}=\mathbf{0}}^{\mathrm{N}} \mathrm{R}_{\max },
$$

where $\mathrm{N}$ is the total number of active rtPS connections.

For nrtPS service flows,

Adaptive quantum value $\left(Q^{t}\right)$ should be in between minimum and maximum thresholds, $\mathrm{Q}_{\mathrm{nrt}}(\min )$ and $\mathrm{Q}_{\mathrm{nrt}}(\max )$ to achieve fairness and efficiency, where

$$
\begin{aligned}
\mathrm{Q}_{\mathrm{nrt}}(\min )= & \sum_{\mathrm{i}=\mathbf{0}}^{\mathrm{N}} \mathrm{R}_{\min } \\
\mathrm{Q}_{\mathrm{nrt}}(\max )= & \sum_{\mathrm{i}=\mathbf{0}}^{\mathrm{N}} \mathrm{R}_{\max }
\end{aligned}
$$

\subsubsection{Quantum for $B E$}

$$
\mathrm{Q}_{\mathrm{be}}=\operatorname{avg}\left(\mathrm{pk} \_\operatorname{size}(\mathrm{be})\right),
$$

\subsubsection{Advantage of RTAS}

No fragmentation headache, still no bandwidth wastage, fairness to all service flows, even for BE.

In [7] and [9] they use fragmentation if current data packet of a specific priority queue is larger than the remaining bandwidth in the TDD frame.

On the contrary, the RTAS scheduler doesn't fragment data packets in such a case, instead, it explores, in an additional round, the other priority queues in order to find a smaller packet that may fit into the frame. In this way bandwidth utilization is maximized while avoiding system complexity due to fragmentation.

\subsubsection{Drawback of RTAS}

Throttled the nrtPS flows

As $\mathrm{Q}^{\mathrm{t}}$ depends directly on queue length, it is limited to $\mathrm{Q}_{\mathrm{nr}}(\mathrm{min})$ and $\mathrm{Q}_{\mathrm{nr}}(\max )$ boundaries for nrtPS flows to avoid unfairness for other priority queues, which results in reduction of throughput of nrtPS flows of RTAS when compared with DFDQ, as shown in the simulation results of [2].
International Journal of Computer Applications (0975 - 8887)

Volume 126 - No.12, September 2015

\subsection{Modified RTAS}

To improve the throughput of nrtPS flows few modifications are proposed in RTAS and are shown in the algorithm by underlining them. As nrtPS needs more bandwidth occasionally, and the delay constrained flows UGS, ertPS, and rtPS are generally not congested and require less bandwidth at regular intervals, hence some more bandwidth can be allocated to nrtPS when it needs and can gain more throughput.

\subsubsection{The main steps of the algorithm(with modifications underlined)}

1) At the beginning of each frame, update an active list, which contains only priority queues that have at least one data packet to send.

2) Calculate real-time quantum values $Q^{t}[i]$, for each class of service ' $\mathrm{i}$ ', using the equations given above./*where $\mathrm{i}=1$ for $\mathrm{UGS}, \mathrm{i}=2$ for ertPS, $\mathrm{i}=3$ for $\mathrm{rtPS}, \mathrm{i}=4$ for $\operatorname{nrtPS}$ and $\mathrm{i}=5$ for $\mathrm{BE}$ service classes $* /$

\section{1) Set Need More Flag(NMF) /* to indicate the high bandwidth requirement of nrtPS */}

If $\left(Q_{\text {nrt }}>\mathbf{Q}_{\text {nrt }}(\max )\right) / *$ calculated quantum value is more than upper limit */

$$
\mathrm{NMF}=1
$$

\section{Else}

$$
\mathbf{N M F}=\mathbf{0}
$$

\section{Endif}

3) Initialize Deficit Counters(DC) to the calculated quantum values: $\mathrm{DC}[\mathrm{i}]=\mathrm{Q}^{\mathrm{t}}[\mathrm{i}]$, for every $\mathrm{i}$,

and available bandwidth (av_bw) to the total frame bandwidth (total_bw): av_bw = total_bw.

4) Fill out data packets into the TDD frame starting from the highest priority queue( UGS,ertPS, rtPS, nrtPS then BE)

If packet size (pk size) is less than remaining bandwidth (pk_size < av_bw) then serve it in the TDD frame.

5) Update deficit counter and available bandwidth after serving each packet as follows:

$$
\begin{aligned}
& \text { DC[i] }=\text { DC }[i]-p k \_s i z e \\
& \text { av_bw }=a v \_b w-p k \_s i z e
\end{aligned}
$$

6) Continue removing packets from the current sub queue until one of the conditions occurs:

$$
\begin{aligned}
& \text { 1) } \mathrm{DC}[\mathrm{i}]<=0 \text { (or) } \\
& \text { 2) priority sub queue gets empty (or) } \\
& \text { 3) frame is full( av_bw }=0 \text { ) }
\end{aligned}
$$

7) If condition 1 or 2 stands, move to next priority queue and repeat from step 4

7.1) If all priority queues are deficit and frame is still not full and width */

if $(\mathrm{NMF}==1)$ and $/ *$ nrtPS flow needs more band

If (UGS and ertPS and rtPS Queues are empty) $/ *$ all high priority flows are not ready now*/

then: 
nrtPS $/ *$ giving one more chance $* /$

1)Update the deficit counter of

i.e., DC[4]=DC[4]+Qn[4] /*to

$\operatorname{nrtPS} * /$

another chance in this turn*/

2) $\mathrm{NMF}=\mathbf{0} / *$ not to give

\section{3)repeat from step 4}

\section{Endif}

8) If all priority queues are deficit, and frame is still not full,

update deficit counters $(\mathrm{DC}[\mathrm{i}]=\mathrm{DC}[\mathrm{i}]+\mathrm{Q}[\mathrm{i}])$,

then move to step 4 for a new round.

9) If the (pk_size > av_bw),

then explore the other non empty priority queues only once, in the objective to search for a smaller packet that can fit into the frame.

10) If condition 3 stands, or smallest pk_size is still larger than the remaining bandwidth in the frame, then send out the frame.

\subsection{Proposed Integration of Modified RTAS with Queue aware Scheduling}

Having the advantage of high throughput achieved with concurrent transmission technique, Queue aware scheduling is one of the best scheduling algorithm, but it was not considering the priorities of the packets. To add the QoS consideration to the high throughput advantage, an Integration of Modified Real Time Adaptive Scheme with Queue aware scheduling algorithm is proposed. The integration process is depicted in Figure 2.

BS maintains a set of five Sub Queues for UGS, ertPS, rtPS, nrtPS and BE service flows for every MS connected to it. The proposed scheme is based on two levels.

In the first level, all the incoming packets are received by the Classifier Module(CM), then they are classified based on their destination MS, type of the service flow, and are redirected to the corresponding Service Class Sub Queue(SCSQ). As specified in Modified RTAS(MRTAS), the packets are inserted into their SCSQs based on the Service Class that it belongs to. That is, the rtPS packets are inserted based on Earliest Deadline First(EDF) mechanism to meet their Delay requirements. All other packets are queued in their respective Queues based on FCFS. Based on the MRTAS algorithm and the Quantum values calculated, the packets are picked from SCSQs and inserted into the main queues of the corresponding MS.

In the second level, Queue aware scheduling algorithm [1] consider the main queues status at BS(BSMQ) and Intermediate queues status at RS(RSIQ). Each RS maintains Intermediate queues for every connected MS. In Queue aware scheduling, Greedy Algorithm prepares Concurrent Transmission Scenarios(CTS) by applying the back pressure flow control mechanism [12] to maximize the end-to-end throughput in multi-hop wireless network. Then the scheduling is done optimistically by finding high throughput solution to the LPP.

The proposed Adaptive Q-aware Scheduling Algorithm with modified RTAS considered QoS requirements of different service flows and maintaining steady throughput when compared with existing algorithms like RTAS and DFPQ and also shown reasonable improvement in throughput of nrtPS flows compared with RTAS.

\section{SIMULATIONS RESULTS}

To compare the performance of the proposed Adaptive Qaware Scheduling algorithm with other scheduling algorithms with respect to QoS and Throughput, the Simulations have been carried out using OPNET 14.5 simulator [15] . The results show that the QoS requirements of rtPS service flow like Packet Delay and Drop rate with Adaptive Queue aware scheduling has been reduced than DFPQ and are in line with RTAS Algorithm. Throughput results of Adaptive Queue aware scheduling show that the performance is nearer to RTAS and better than DFPQ for rtPS service flows, it is better than RTAS and nearer to DFPQ for nrtPS service flows, which shows the affect of the Need More Flag(NMF) modification of MRTAS. Throughput of BE class of Adaptive Queue aware scheduling is in line with RTAS and DFPQ.

The basic simulation setup consists of 1 Base Station, 3 Relay Stations, and 25 Mobile Stations. The MS are deployed randomly in a cell with radius $1 \mathrm{~km}$, and random way point model is used for MS's mobility with moving speed randomly chosen from 0 to $5 \mathrm{~m} / \mathrm{s}$. The $3 \mathrm{RS}$ are placed in fixed positions, with distance from BS to each RS set to $600 \mathrm{~m}$, and the angle between two neighboring RS set to degree $120^{\circ}$. The assumed TDD frame for DL channel with frame bandwidth is $100 \mathrm{Kbps}$ and frame duration is $20 \mathrm{~ms}$. Traffic is generated in BS with various traffic rates and the packet arrival is described by Batch Markovian Ariival Process(BMAP)

In this paper, the focus is on the priorities of the packets arriving at the main Queues at BS in Q-aware scheduling algorithm, and proposed an integration of MRTAS with Queue aware scheduling algorithm. The Proposed Adaptive Q-aware scheduling algorithm performance is compared with Q-aware, DFPQ and RTAS. As UGS and ertPS flows are allocated fixed bandwidth grants at the beginning of each frame, only the traffic from rtPS, nrtPS and BE service classes is considered. These three classes are considered with three different connections, each with different average packet sizes (pk_size), Minimum Reserved Rate $\left(\mathrm{R}_{\min }\right)$ and Maximum Sustained Rate $\left(R_{\max }\right)$. Real time quantum values $\left(Q^{t}\right)$, are calculated based on the corresponding equations for rtPS, nrtPS and BE. It is assumed that rtPS connections violate the maximum sustained rate at the second minute of simulation time with an average of $10 \%$ and until the end of the simulation. 




Fig 2: Adaptive Queue aware scheduling

\subsection{QoS Consideration}

The impact of various queues at BS and RSs and the issue of fragmentation also effects the packet delay and drop rate.

\subsubsection{Packet Delay}

Packet delay is observed with respect to DFPQ, RTAS and AQSA for rtPS. As shown in figure 3 the average delay is about $99 \mathrm{~ms}$ with DFPQ, 29ms with RTAS and $31 \mathrm{~ms}$ with AQSA.

\subsubsection{Packet Drop Rate:}

The packet drop rate is about 73kbps with DFPQ and is almost negligible with RTAS and AQSA as shown in figure 4. This is because of the consideration of current queue sizes in calculating the Quantum values of the service flows. It is also observed that the channel utilization is nearly $100 \%$ with DFPQ because of the fragmentation and is around $98 \%$ in RTAS and $97 \%$ in AQSA even without fragmentation. 


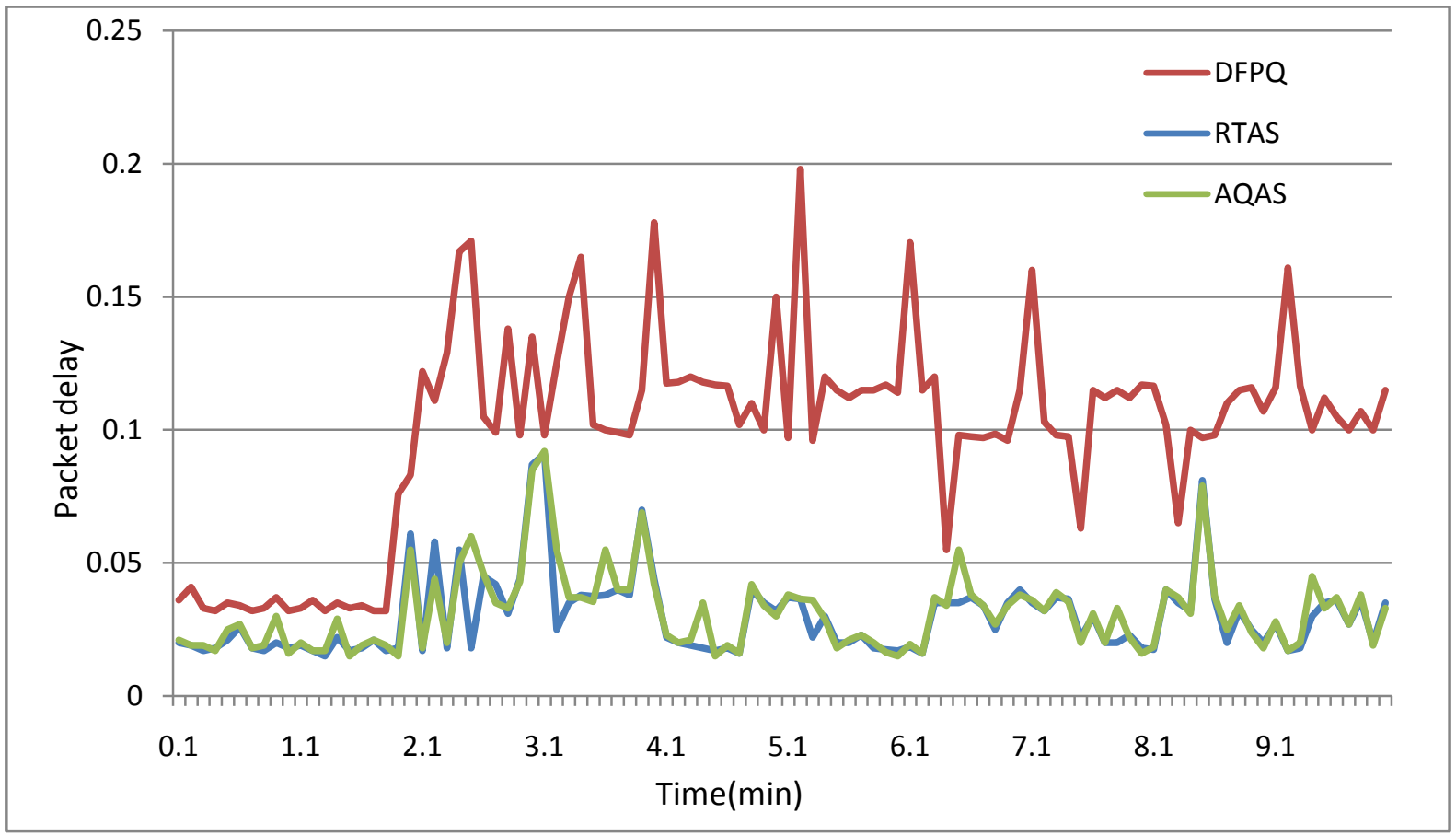

Fig 3: Comparison of Packet Delay of rtPS flows with DFPQ, RTAS and AQSA

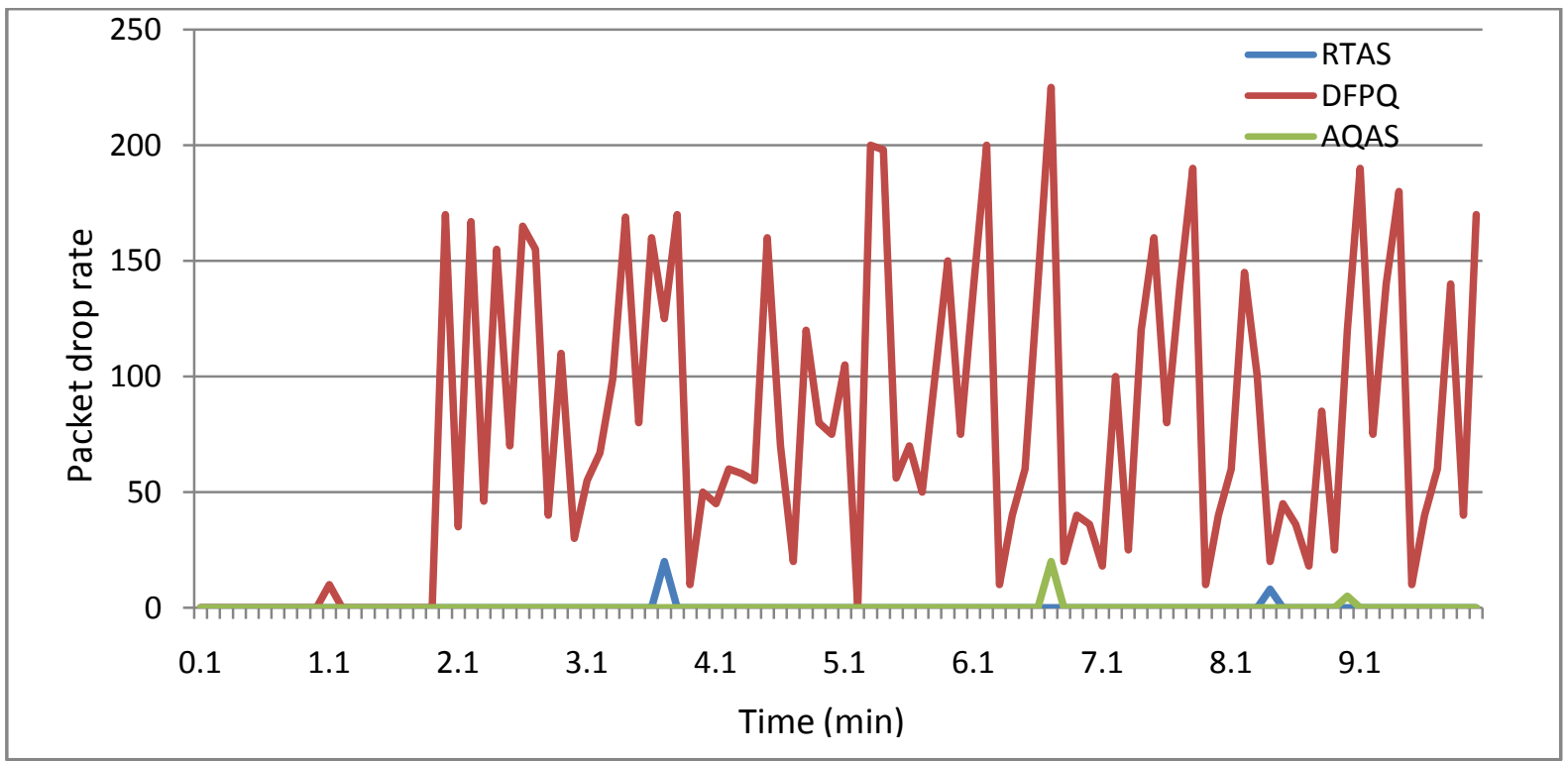

Fig 4: Drop rate for rtPS w.r.t. DFPQ, RTAS and AQSA

\subsection{Throughput Comparison}

In Figure 5, the throughput achieved, for rtPS using proposed Adaptive Q-aware scheduling algorithm (AQSA) with RTAS and DFPQ is compared.

It is observed that all the three schedulers have nearly similar throughput for the three classes of traffic at the beginning of the simulation and until the second minute of simulation time, at which rtPS flows exceed their Rmax and this results in a considerable effect on the throughput for the whole network. After the second minute, DFPQ raises rtPS flows throughput to a maximum level and continues to serve nearly the same amount of rtPS data packets in each frame, while RTAS achieves higher throughput for rtPS, and AQSA performs equally well with RTAS for rtPS. 


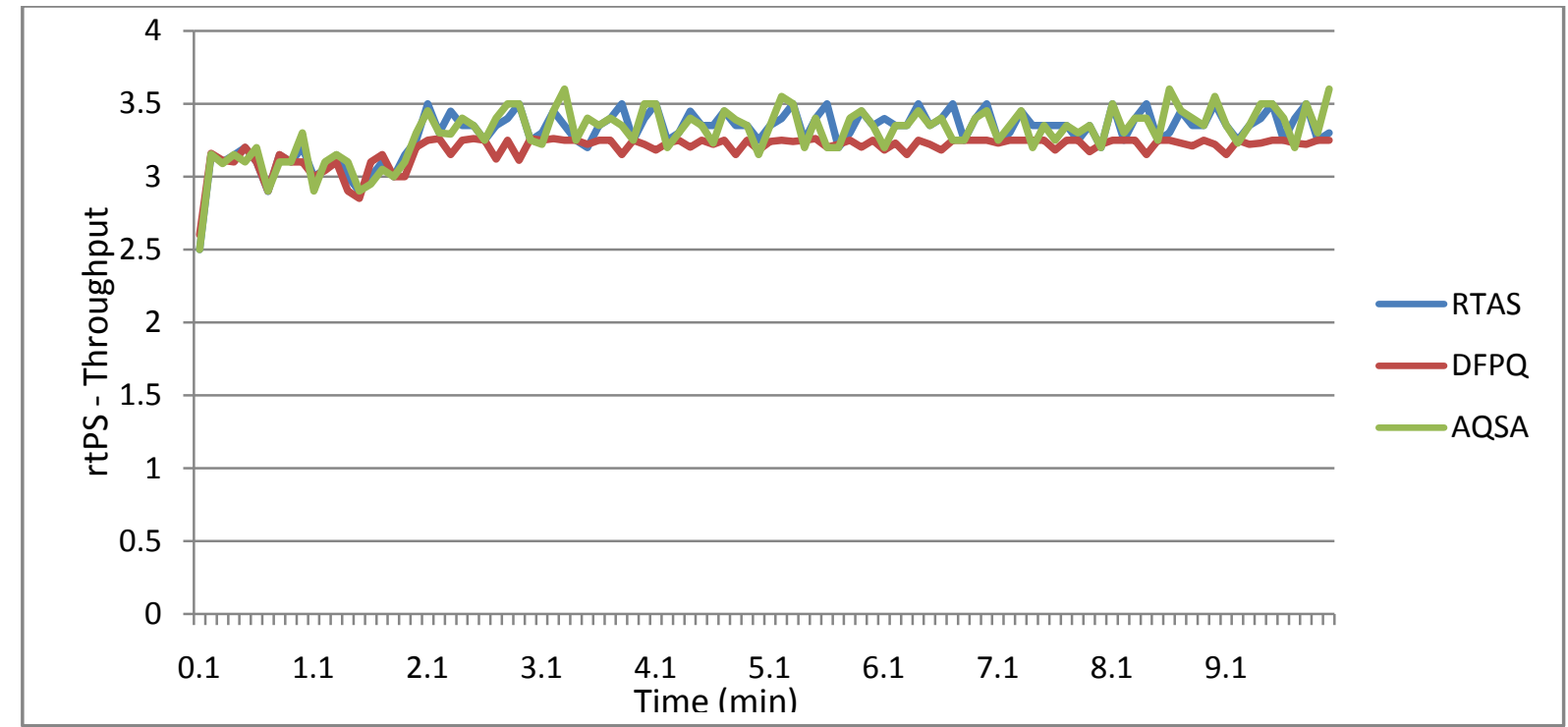

Fig 5: Throughput of rtPS class with DFPQ, RTAS and Modified RTASand AQSA

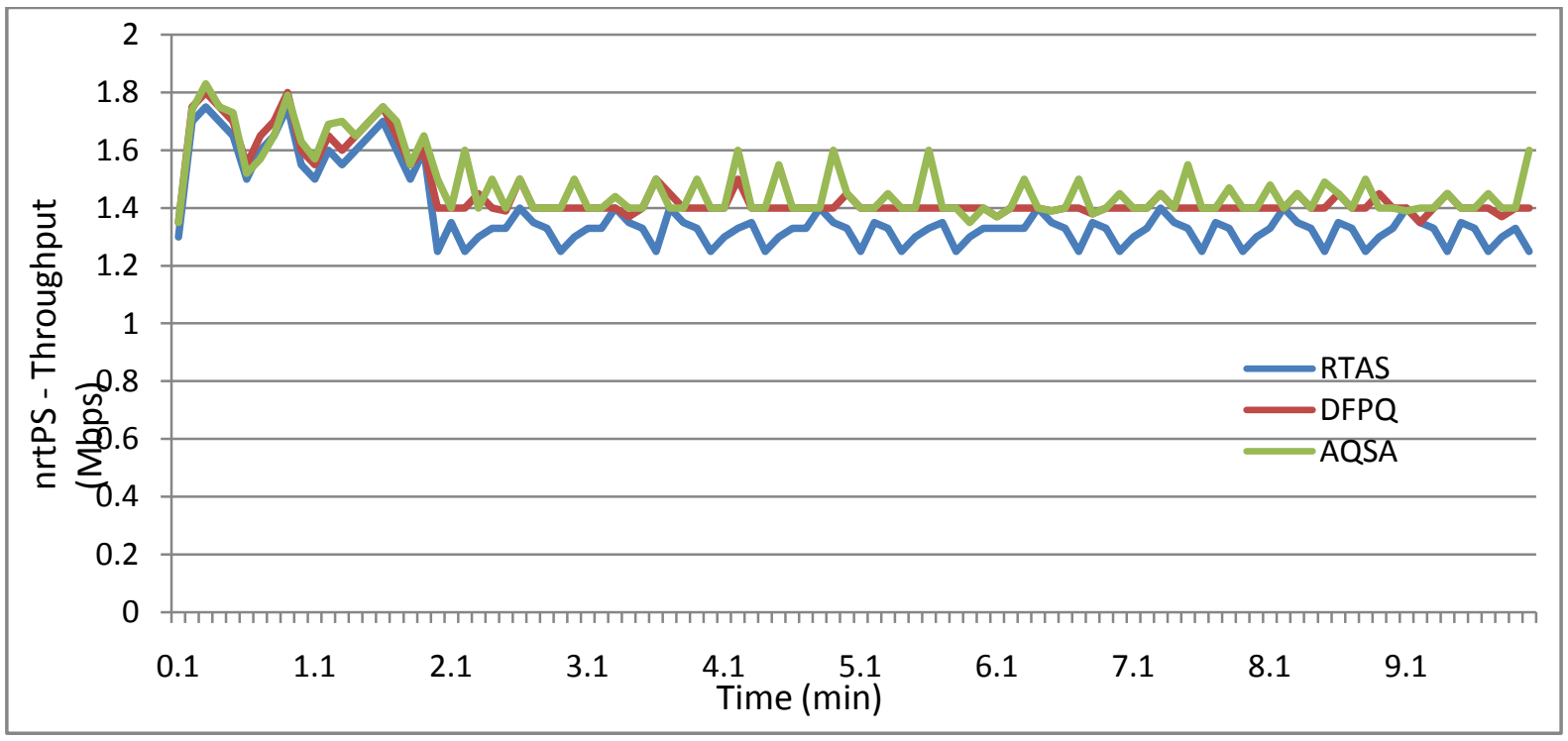

Fig 6: Throughput of nrtPS class with DFPQ, RTAS and AQSA

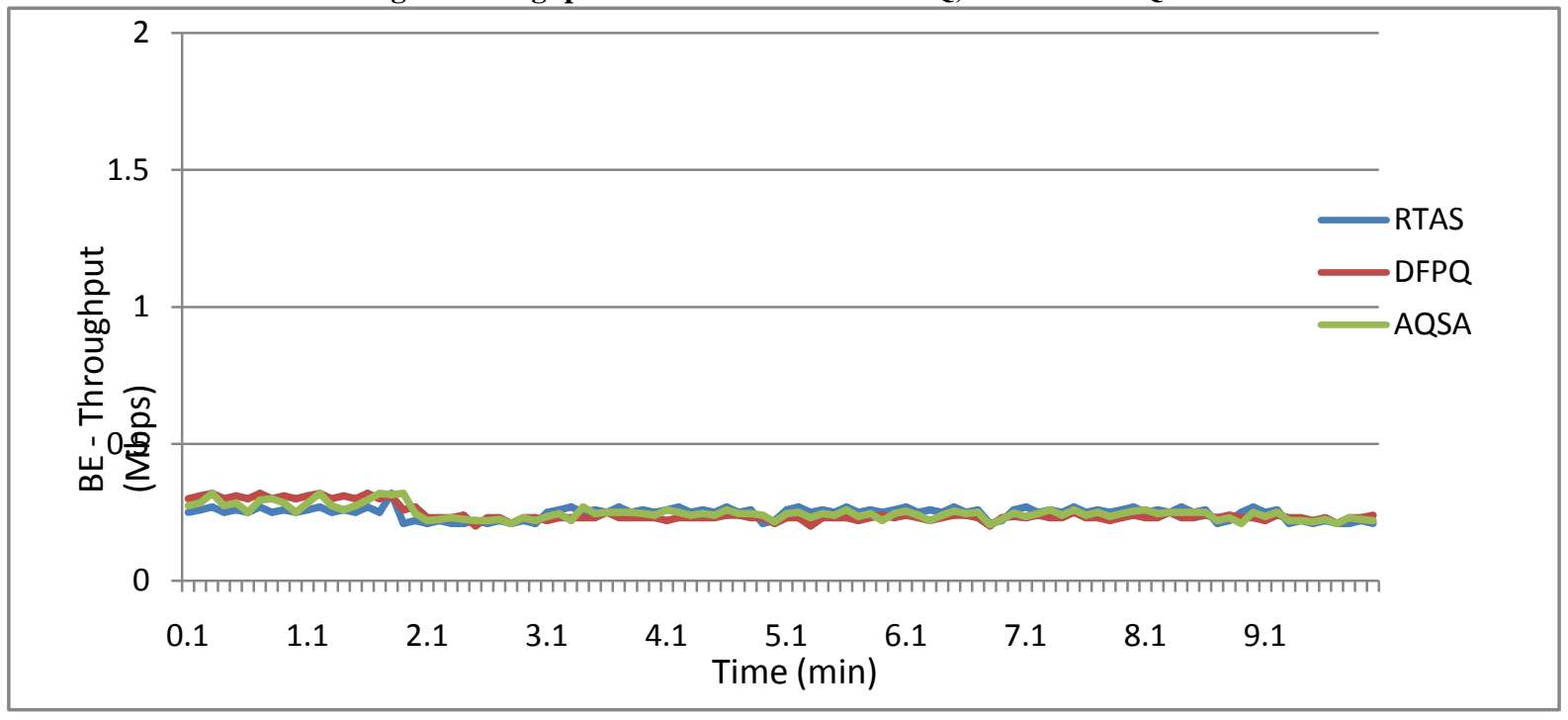

Fig 7: Throughput of BE class with DFPQ, RTAS and AQSA 
Whereas from figure 6, RTAS observes less throughput for nrtPS comparing with DFPQ, but AQSA records nearly equal performance with DFPQ, which is a result of the Need More Flag(NMF) modification in MRTAS. From figure 7, it can be observed that the throughput performance of all the three schedulers are almost similar for BE class.

\section{CONCLUSION \& FUTURE WORK}

In this paper, an integration of Modified Real Time Adaptive Scheduling Scheme with Queue-aware Scheduling Algorithm is proposed for IEEE 802.16j networks to achieve high throughput while considering the QoS requirements of different service flows. In the first level of Adaptive Scheduling Scheme, the QoS requirements of different service flows are considered. In the second level, the Queue aware scheduling prepares Concurrent Transmission Scenarios (CTS) in consideration of high back pressure links and then allocates the frames to the slots in an optimal way so as to maximize the overall throughput by considering concurrent transmissions and current queue sizes at BS and RSs, hence achieved high throughput when compared with other Down Link Scheduling Algorithms. The proposed integration of Modified Real Time Adaptive Scheme with Queue aware scheduling assigns the optimal resources to high priority traffic without penalizing the low priority traffic. In future, the throughput efficiency of Queue aware scheduling algorithm can be evaluated in the context of Directional Antennas.

\section{REFERENCES}

[1] H. Chen, X. Xie and H. Wu, "A Queue-aware Scheduling Algorithm for Multihop Relay Wireless Cellular Networks", IEEE Mobile WIMAX Symposium, 2009.

[2] Ghazal S, Mokdad L and Ben-Othman J, "A Real Time Adaptive Scheduling Scheme for Multiservice Flows in WiMAX networks", IEEE GLOBECOM, 2008

[3] IEEE Standard for Local and Metropolitan Area Networks-Part 16: Air Interface for Fixed Broadband Wireless Access SystemsAmendment: Multihop Relay Specification, 2007, IEEE802.16j. [Online]. Available: http://www.ieee802.org/16/

[4] IEEE Standard 802.16e,'IEEE Standard for Local and metropolitanarea networks Part 16: Air Interface for Fixed and Mobile Broadband Wireless Access Systems Amendment 2: Physical and Medium Access Control Layers for Combined Fixed and Mobile Operation in $\begin{array}{cccc}\text { Licensed } & \text { Bands",February } 2006 & \text { [Online]. } \\ \text { Available: } & \text { http://www.iee802.org/16/ }\end{array}$

[5] IEEE 802.11: Wireless LAN Medium Access Control(MAC) and Physical Layer (PHY) Specifications, IEEE -SA . 5 April 2012. doi: 10.1109/IEEESTD.2012.6178212 [Online].

Available: http://www.ieee802.org/11/

[6] H. Viswanathan and S. Mukherjee, "Throughput-Range Tradeoff of Wireless Mesh Backhaul Networks," IEEE J. Sel. Areas Commun., vol. 24, no. 3, pp. $593-$ 602, Mar. 2006.

[7] J. Chen, W. Jiao, H. Wang, "A Service Flow Management Strategy for IEEE 802.16 Broadband Wireless Access System",. In proc. of IEEE ICC 2005, Seoul, Korea (May 2005)

[8] K. wongthavarawat, A. Ganz,"Packet scheduling for QoS support in IEEE 802.16 broadband wirelessaccess systems", international journal of communication system 2003

[9] H. Safa, and al., "New Scheduling Architecture for IEEE 802.16 Wireless Metropolitan Area Network", AICCSA, May 2007.

[10] N.Ruangchaijatupon, Y.JI, ”A traffic-based adaptive deficit scheduling for QoS support in IEEE 802.16e TDD mode",ICNS'07, 2007

[11] S.J. Lin, W. H. Sheen, I.K. Fu, and C.C. Huang, "Resource Scheduling with Directional Antennas for Multihop Relay Networks in Manhattan-like Environment," in Proc. IEEE Mobile WiMAX Symp., 2007, pp. 108-113.

[12] L. Tassiulas and A. Ephremides, "Stability properties of constrained queueing systems and scheduling policies for maximum throughput in multihop radio networks," IEEE Trans. Auto. Ctrl., vol. 37, no. 12, pp. 1936-1948, Dec. 1992.

[13] M. Shreedhar, G.Varghese, "Efficient Fair Queuing Using Deficit Round Robin", IEEE/ACM Transactions on Networking, June 1996.

[14] Georgiadis L, Guerin R, Parekh A, "Optimal Multiplexing on a Single Link: Delay and Buffer Requirements", Proceedings of IEEE INFOCOM94, vol.2, p524532, 1994.

[15] http://www.opnet.com/university_program/itguru_acade mic edition/. 